\title{
ON INVERTIBLE BIMODULES AND AUTOMORPHISMS OF NONCOMMUTATIVE RINGS
}

\author{
ROBERT M. GURALNICK AND SUSAN MONTGOMERY
}

\begin{abstract}
In this article, we attempt to generalize the result that for a commutative ring $R$ the outer automorphism group of $R$-automorphisms of $M_{n}(R)$ is abelian of exponent $n$. It is shown that a slightly weaker stable version of the result is still valid for affine semiprime noetherian pi rings. We also show that the automorphism group of an affine commutative domain of positive dimension acts faithfully on the spectrum of the domain. We investigate other questions involving bimodules and automorphisms and extend a result of Smith on the first Weyl algebra as a fixed ring.
\end{abstract}

\section{INTRODUCTION}

Let $R$ be a ring with 1 . In this article we are interested in comparing the automorphism group of $M_{n}(R)$, the ring of $n \times n$ matrices over $R$, and the automorphism group of $R$. In particular, if $R$ is commutative, it is a classical result that the outer automorphism group of $R$-automorphisms of $M_{n}(R)$ is an abelian group of exponent dividing $n$. This can be restated in terms of invertible bimodules. The result is equivalent to the easy fact that any projective rank one module $X$ with $n X$ free over a commutative ring has order dividing $n$ in the Picent group of $R$-see [RZ and KO]. In the noncommutative case, there is still a well known relationship between automorphisms and the Picard group of invertible bimodules.

In this paper we obtain analogs of the commutative result modulo stably free modules. One of the key ideas is to study the Picent group by considering a suitable normal subgroup (the subgroup which commutes with primes). The normal subgroup and the quotient group have very different structures. The behavior of the normal subgroup (at least stably) resembles the commutative case. There is no analog of the quotient in the commutative case. Our best results in this direction are (see Lemma 3.2, and Theorems 3.6, 4.1, and 4.2):

Theorem A. Let $k$ be a field or the integers. Let $R$ be a semiprime noetherian affine pi ring of Krull dimension $d$ and pi degree $f$. Let $G=\operatorname{Picent}_{n}(R)$ be the group of central invertible $R$-bimodules such that the direct sum of $n$ copies of any element is a free left $R$-module. Let $G_{1}$ be the (normal) subgroup of $G$

Received by the editors January 2, 1992 and, in revised form, January 30, 1992.

1991 Mathematics Subject Classification. Primary 16A38, 16A72, 11R65; Secondary 16A64, 16 A74.

The first author was partially supported by NSF grant DMS 8901492 . The second author was partially supported by NSF grant DMS 8901491. 
consisting of those elements which commute with all primes of $R$. Then there exists a normal subgroup $G_{2}$ of $G$, with $G_{2} \subseteq G_{1}$ such that:

(i) Every element of $G_{2}$ is stably free as a left $R$-module.

(ii) $G / G_{1}$ is a subgroup of a finite direct product of symmetric groups of degree $f$.

(iii) $G_{1} / G_{2}$ is nilpotent of class at most $d$ and has exponent dividing $n^{u}$, where $u$ is the least positive integer bigger than $\log _{2} d$.

In particular, $G / G_{2}$ is locally finite of bounded exponent.

The previous theorem yields results about automorphisms of matrix rings. We state a special case here.

Corollary B. Let $R$ be as in Theorem A. Assume that every invertible R-bimodule which is stably free as a left $R$-module is in fact free as a left $R$-module. If $\alpha$ is a central automorphism of $M_{n}(R)$, then $\alpha^{n^{u}} f !=\beta \gamma$, where $\beta$ is an extension of an automorphism of $R$ and $\gamma$ is inner.

In general, one would have to allow $\beta$ to be induced by an invertible bimodule which is stably free as a left module.

The proof of Theorem A depends on proving a nilpotence result for certain elements of $K_{0}$. This is a result of Bass (see [W]) in the commutative case. This result is generalized in [G3] with a somewhat different proof. We also need a result that relates the action of an automorphism on the center of $R$ and on Spec $R$. In particular, we show that if $R$ is a commutative affine domain of positive dimension over a field $k$, then $\operatorname{Aut}_{k}(R)$ acts faithfully on $\operatorname{Spec} R$ (Theorem 6.3). This uses a result of $[F]$ as extended by Braun and Vonessen [BV] which reduces the problem to finite groups. Finally, we need a result of Braun [B] about primes of the trace ring of a prime noetherian pi ring.

We construct examples of rings to show that the results in Theorem A cannot be improved much. In particular, we show the following (see Theorem 5.6).

Example C. Let $k$ be a field admitting a cyclic extension of degree $n$. Then there exists a one dimensional affine noncommutative domain $R$ module finite over its center such that $\operatorname{Picent}_{n}(R)$ contains a subgroup $H$ isomorphic to $P G L_{n}(k)$. All elements of $H$ are stably free as left and right modules. In particular, if $k$ is not algebraic over a finite field, there exist invertible bimodules $X$ with $n X$ free as a left module and $X$ stably free as a left and right module but no power of $X$ is free.

The above also provides an example of a central automorphism of $M_{n}(R)$ such that no power is induced by an automorphism of $R$. We also construct orders $R$ over the integers which have invertible bimodules $X$ which are stably free but not free as left modules. This answers a question of Gustafson and Roggenkamp [GR2, Question 4.10].

We also give examples to show that even stably the Picent group of $R$ need not be abelian (or even solvable). Indeed, we show (see Theorem 4.3).

Example D. Let $k$ be a field and $n>3$. There exists a prime noetherian affine $k$-algebra of pi-degree $2 n$ and Krull dimension 2 such that (in the notation of Theorem A), $G / G_{1}$ is a symmetric group of degree $n$ and all stably free elements of $\operatorname{Picent}(R)$ are contained in $G_{1}$. 
In a different direction, although still in the spirit of relating bimodules and automorphisms, we extend a result of Smith on the Weyl algebra as a fixed ring. In particular, we prove

Theorem $\mathbf{E}$. Let $A$ be a simple noetherian domain satisfying the following conditions:

(i) $k=Z(A)$ is algebraically closed.

(ii) Every unit of $A$ is central.

(iii) If $X$ is a torsion element of $\operatorname{Picent}(A)$, then $X$ is a free left $R$-module.

Then there exists no $k$-domain $S$ such that $S^{G}=A$ where $G$ is a finite nonperfect subgroup of $\operatorname{Aut}_{k}(S)$ and $|G| \neq 0$ in $k$.

Applying this result to the first Weyl algebra (see Corollary 7.3), we show that the first Weyl algebra is not the fixed ring of a finite nonperfect group acting on a domain.

This article is organized as follows. In $\S 2$, we discuss the relationship between automorphisms and invertible bimodules. In $\S 3$, we prove various finiteness results for affine pi rings. In $\S 4$, we analyze the group structure of the Picent group more closely. In $\S 5$, we consider one dimensional rings and construct an example to show stably free cannot be replaced by free in the results of $\S 3$. In $\S 6$, we include some unpublished results of Braun and Vonessen which we extend on automorphisms of commutative affine domains and pi rings. In the final section, we consider the fixed ring problem.

The authors would like to thank A. Braun and N. Vonessen for several interesting discussions on this problem and for allowing us to include some of their results.

\section{Preliminary Results}

(2.1) We need some definitions. Let $\mathscr{I}$ be the Picard group of invertible bimodules of $R$. The group operation is tensor product. Unless otherwise stated all isomorphisms refer to left modules. Let $Z=Z(R)$ be the center of $R$. Let
(a) $\mathscr{I}_{n}=\{X \in \mathscr{I} \mid n X \cong n R\}$,
(b) $\mathscr{I}^{s f}=\{X \in \mathscr{I} \mid X$ is stably free $\}$,
(c) $\mathscr{J}=\{X \in \mathscr{I} \mid P X=X P \forall P \in \operatorname{Spec} R\}$,
(d) $\mathscr{J}_{n}=\mathscr{J}_{n} \cap \mathscr{J}$,
(e) $\mathscr{J}^{s f}=\mathscr{I} s f \cap \mathscr{J}$,
(f) $\operatorname{Picent}(R)=\{X \in \mathscr{I} \mid z x=x z \forall x \in X, z \in Z\}$,
(g) $\operatorname{Picent}_{n}(R)=\operatorname{Picent}(R) \cap \mathscr{I}_{n}$,
(h) $\mathscr{C}=\left\{X \in \mathscr{I} \mid X\right.$ and $X^{-1}$ are isomorphic to ideals of $\left.R\right\}$,
(i) $\mathscr{S}=\mathscr{C} \cap \mathscr{I}^{s f}$, and
(j) $\mathscr{C}_{n}=\mathscr{C} \cap \mathscr{I}_{n}$.

Lemma 2.2. The subsets of $\mathscr{I}$ defined above except in (b) and (i) are all subgroups of $\mathscr{I}$. The subsets defined in (b) and (i) are subgroups whenever $R$ has a homomorphic image contained in a noetherian ring.

Proof. Suppose $X, Y \in \mathscr{I}_{n}$. So $n Y \cong n R$ as left modules. Tensor on the left with $X$. This implies that $n(X \otimes Y) \cong n X \cong n R$ as left modules. Similarly, 
by tensoring with $Y^{-1}$, we see that $n Y^{-1} \cong n R$. Thus $\mathscr{J}_{n}$ is a subgroup. The proof for (b) is essentially the same. The remaining cases are trivial.

We are particularly interested in $\mathscr{I}_{n}$ because of its connection with automorphisms of $M_{n}(R)$. Recall that if $\sigma$ is an endomorphism of $R$, then we can define the $R$-bimodule $X={ }_{1} R_{\sigma}$ by taking $X=R$ as left modules and letting $r \in R$ act on the right via $\sigma(r)$. We record some trivial properties of these bimodules (see [CR, §55]).

Lemma 2.3. (a) If $X$ is an R-bimodule which is isomorphic to $R$ as a left module, then $X \cong{ }_{1} R_{\sigma}$ for some endomorphism $\sigma$ of $R$.

(b) ${ }_{1} R_{\rho} \otimes{ }_{1} R_{\sigma} \cong{ }_{1} R_{\rho \sigma}$ as bimodules.

(c) ${ }_{1} R_{\sigma}$ is invertible if and only if $\sigma$ is an automorphism of $R$.

(d) ${ }_{1} R_{\sigma} \cong{ }_{1} R_{1}=R$ if and only if $\sigma$ is an inner automorphism of $R$.

Proof. (a) If $X=R \alpha$, define $\sigma$ by $\sigma(r) \alpha=\alpha r$. Clearly $X \cong{ }_{1} R_{\sigma}$.

For (b), note that the isomorphism is given by the map $a \otimes b \rightarrow a \rho(b)$. Now (c) follows from (b) and Lemma 2.2. (d) is a straightforward observation.

Lemma 2.4. If $X \in \mathscr{I}_{n}$, then there exists $\alpha \in M_{n}(X)$ such that $M_{n}(X)=$ $\alpha M_{n}(R)=M_{n}(R) \alpha$. Consequently, $\mathscr{J}_{n}=\{X \in \mathscr{I} \mid n X \cong n R$ as right $R$ modules\}.

Proof. Note that $M_{n}(X)$ is a rank one free left $M_{n}(R)$-module which is an invertible $M_{n}(R)$-bimodule. The result now follows by applying the previous result to $M_{n}(X)$.

By Lemmas 2.3 and 2.4, if $X \in \mathscr{I}_{n}$, then $M_{n}(X)=\alpha M_{n}(R)=M_{n}(R) \alpha$ and $X$ induces an automorphism of $M_{n}(R)$ given by $r \rightarrow s$, where $r \alpha=\alpha s$. If a different generator for $M_{n}(X)$ is chosen, then the automorphism will change by an inner automorphism of $M_{n}(R)$. Thus we have homomorphisms

$$
\phi: \mathscr{I}_{n} \rightarrow \text { Out } M_{n}(R),
$$

$$
\phi^{\prime}: \mathscr{I}_{n} \rightarrow \text { Aut } Z \text {. }
$$

Clearly $\operatorname{ker}\left(\phi^{\prime}\right)=$ Picent $_{n} R$. In fact, $\phi^{\prime}$ can be extended to $\mathscr{I}$ [CR, 55.7]. We note the following

Proposition 2.6. $\phi$ is an isomorphism of $\mathscr{I}_{n}$ and Out $M_{n}(R)$.

Proof. Obviously $\phi$ is a homomorphism. If $M_{n}(X) \cong M_{n}(R)$ as $M_{n}(R)$ bimodules, then clearly $X \cong R$ as $R$ bimodules. Thus $\phi$ is injective. If $\delta$ is an automorphism of $M_{n}(R)=T$, set $Y={ }_{1} T_{\delta}$. Let $E$ be the idempotent matrix with a 1 in the $(1,1)$ entry and all other entries zero. Then $X=E Y \delta(E)$ is an invertible $R$ bimodule. It is straightforward to verify that $n X \cong n R$ as both left and right modules. Thus $X \in \mathscr{I}_{n}$ and obviously $M_{n}(X) \cong Y$ as $M_{n}(R)$-bimodules. Thus $\phi$ is surjective and the result follows.

The usual results about automorphisms of matrices over commutative rings follow easily. Let $\operatorname{Aut}_{Z}(R)$ denote the group of automorphisms of $R$ which are trivial on the center. Note that there is an obvious embedding of $\operatorname{Aut}(R)$ into $\operatorname{Aut}\left(M_{n}(R)\right)$. Set $\operatorname{Out}_{Z}(R)=\operatorname{Aut}_{Z}(R) / \operatorname{Inn}(R)$. 
Corollary 2.7. (a) $\operatorname{Out}_{Z}\left(M_{n}(R)\right) \cong \operatorname{Picent}_{n} R$.

(b) If $R=Z$ is commutative, then $\operatorname{Out}_{R}\left(M_{n}(R)\right)$ is abelian of exponent dividing $n$.

Proof. The first statement is an immediate consequence of Proposition 2.6. If $R$ is commutative, then $\operatorname{Picent}(R)$ is an abelian group. If $X \in \operatorname{Picent}_{n} R$, then $X^{n} \cong \wedge^{n}(n X) \cong \wedge^{n}(n R) \cong R$. Thus Picent $_{n} R$ is abelian of exponent dividing $n$, as desired.

There is a version of the commutative result that extends to arbitrary rings.

Corollary 2.8. Identify $R$ with the subring of scalars of $M_{n}(R)$. Let

$$
G=\operatorname{Aut}_{R}\left(M_{n}(R)\right) / \operatorname{Inn}_{R}\left(M_{n}(R)\right)
$$

be the group of automorphisms which are trivial on $R$ modulo the inner automorphisms which are trivial on $R$. Then $G$ is abelian of exponent $n$.

Proof. Note that the centralizer of $R$ in $M_{n}(R)$ is just $M_{n}(Z)$. Thus $M_{n}(Z)$ is $G$-invariant. Since $M_{n}(R)$ is generated by $R$ and $M_{n}(Z)$, the restriction map from $\operatorname{Aut}_{R}\left(M_{n}(R)\right)$ to $\operatorname{Aut}_{Z}\left(M_{n}(Z)\right)$ is injective. By Corollary 2.7(b), it follows that the $n$th power of any automorphism is inner (so given by conjugation by an element of $\left.G L_{n}(Z)\right)$. The same is true for the commutator of any two such elements. The result follows.

We close this section with the following observation.

Corollary 2.9. $\mathscr{I}_{n}$ is a normal subgroup of $\mathscr{I}_{n}$.

Proof. By (2.5.1), each element of $\mathscr{I}_{n}$ induces an automorphism of $\operatorname{Spec} M_{n}(R)$ and so a permutation of $\operatorname{Spec} R$. The kernel of this permutation representation is $\mathscr{J}_{n}$.

In fact, it is not difficult to show that $\mathscr{I}$ has a permutation representation on the ideals of $R$. (The representation maps $X$ to the permutation $I \rightarrow X^{-1} I X$.) Since this permutation preserves products of ideals, it sends primes to primes. Thus in fact $\mathscr{J}$ is normal in $\mathscr{I}$.

\section{Torsion in the Picard group}

We want to extend as far as possible the classical results about automorphisms of $M_{n}(R)$ for $R$ commutative (Corollary 2.7(b)). Note that $\mathrm{Out}_{Z}(R)$ naturally embeds in $\operatorname{Out}_{Z}\left(M_{n}(R)\right)$. One might conjecture that the quotient group is abelian and of exponent dividing $n$. Unfortunately, neither result is true. The subgroup need not be normal and outer automorphisms of infinite order can arise. We consider such examples in $\S 5$.

However, for certain rings we do obtain an analog of the torsion result.

The following result will be useful in proving certain elements of $\operatorname{Pic}(R)$ have finite order modulo the stably free elements.

Lemma 3.1. Let $R$ be a ring. Let $T=K_{0}(\mathscr{B})$, where $\mathscr{B}$ is the category of $R$ bimodules which are finitely generated projective on the left and right. Note $T$ is a ring (with multiplication being tensor product). Let $U=K_{0}(R)$ (left modules). Then $U$ is a left $T$-module. Let $X$ be a projective bimodule (i.e., left and right projective). If $n X \cong n R$ as left modules for some positive integer $n$ and $(X-R)^{d+1} \cdot R=0$ in $U$ for some nonnegative integer $d$, then $\left(X^{m}-R\right) \cdot R=0$ 
in $U$ for $m=n^{u}$ for any positive integer $u>\log _{2}(d)$. (i.e., $X^{m}$ is stably isomorphic to $R$ as a left module.)

Proof. Consider the subring $A=Z[X]$ of $T$. Let $I$ be the annihilator of $R$ (considered as an element of $U$ ) in $A$. Set $B=A / I$. Let $t$ be the image of $X$ in the ring $B$. By our assumptions, $(t-1)^{d+1}=0=n(t-1)$. Set $y=(t-1)$. Then $B=\langle 1\rangle+\langle y\rangle+\cdots+\left\langle y^{d}\right\rangle$, as abelian groups.

We claim that for any nonnegative integer $e$, there exists a $b \in B$ such that

$$
t^{n^{e}}=1+b y^{2^{e}} .
$$

Since $n y=0$, the result follows by raising both sides of (3.1.1) to the $n$th power and inducting on $e$. In particular, if $u>\log _{2}(d)$, then (3.1.1) implies $t^{n^{u}}=1$, and the result follows.

For the rest of this section, fix a commutative noetherian domain $k$. Let $R$ be a pi $k$-algebra. We only consider $R$-bimodules which are $k$-central (that is $k$ acts the same on the left and right). Note that when $R$ is semiprime with only a finite number of minimal primes, then $R$ has a semisimple Artinian classical quotient ring $Q$ which is a central localization. We write this as $Q=R Z^{-1}$, although we are only inverting the regular elements of $Z$. In particular, this will apply when $R$ is affine over $k$ and semiprime.

We are mostly interested in central automorphism and correspondingly elements of Picent $R$. However, we prefer to use $\mathcal{J}$ since it is better behaved under homomorphic images. We shall see that the Picent group and $\mathscr{J}$ are closely related. Indeed, we prove that if $k$ is a field and $R$ is semiprime (and not algebraic over $k$ ), then $\mathscr{J}_{n}$ is a (normal) subgroup of $\operatorname{Picent}_{n}(R)$ (Theorem 3.3) and that if $R$ is also noetherian, then the quotient group is of bounded exponent (Theorem 3.5).

Lemma 3.2. Let $R$ be semiprime pi with finitely many minimal primes, and let $X \in \mathscr{I}$. Then the following are equivalent:

(a) $X \in \operatorname{Picent}(R)$.

(b) $X \cong J$ for some $R$-subbimodule of $Q$ as bimodules.

(c) $X \cong I$ for some ideal I of $R$ (as bimodules).

(d) There exist $R$-bimodule monomorphisms $\alpha: R \rightarrow X$ and $\beta: X \rightarrow R$ such that $\alpha \beta$ and $\beta \alpha$ are multiplication by a regular element $c \in Z$.

Proof. Clearly (d) implies (c), (c) implies (b), and (b) implies (a). So we need only prove that (a) implies (d).

Now $Y=X \otimes Q$ becomes a $Q$-bimodule via $r z^{-1}(y \otimes q)=r y \otimes z^{-1} q$ since $X$ is $Z$-central. Since $Y$ is an invertible $Q$-bimodule and $Q$ is semisimple artinian, it follows that $Y \cong Q$ as left modules. Thus $Y \cong{ }_{1} Q_{\rho}$ for some automorphism $\rho$ of $Q$. Since $X$ is $Z$-central, so is $Y$. Thus $\rho$ is trivial on the center of $Q$. Hence by the Skolem-Noether theorem, $\rho$ is inner on $Q$. Thus $Y \cong Q$ as bimodules. Since $X$ is a bisubmodule of $Y$, we have a bimodule embedding of $X$ in $Q$. So we can assume that $X$ is contained in $Q$. Since $X$ is finitely generated as an $R$-module, there exist regular elements $\alpha, \beta \in Z$ such that $\alpha X \subseteq R$ and $\beta R \subseteq X$. Take $c=\alpha \beta$. Now (d) follows.

Theorem 3.3. Let $R$ be a semiprime pi affine k-algebra.

(a) If $R / P$ is not algebraic over $k$ for any minimal prime $P$, then $\mathscr{J}_{n} \subseteq$ $\operatorname{Picent}(R)$. 
(b) If $k$ is the ring of rational integers and $R / P$ has positive Krull dimension for each minimal prime $P$, then $\mathscr{J}_{n} \subseteq \operatorname{Picent}(R)$.

(c) If $X \in \mathscr{J}_{n}$, then some power of $X$ is in $\operatorname{Picent}(R)$.

Proof. Choose $X \in \mathscr{J}_{n}$. We can replace $R$ by $M_{n}(R)$ (and $X$ by $M_{n}(X)$ ) and assume that $n=1$. So $X=R \alpha=\alpha R$, for some $\alpha \in X$ and as above $X \cong{ }_{1} R_{\sigma}$ for some automorphism $\sigma$ of $R$. Since $X \in \mathcal{J}, \sigma$ is trivial on $\operatorname{Spec} R$.

We first prove (a). It suffices to show $\sigma$ is trivial on the center of $R$. Since $R$ has only finitely many minimal primes, it suffices to assume $R$ is prime. By localizing at the nonzero elements of $k$, we can assume that $k$ is a field. Since $R$ is not algebraic over $k$, it has positive Krull dimension. The result now follows by Theorem 6.4 .

The argument of the previous paragraph also proves (b) uniess $R$ is algebraic over $k$. Then $R[1 / b]$ is integral over $k[1 / b]$ for some integer $b$. By the Čebotarev density theorem (or see [J1] for an elementary argument), there are infinitely many primes of $k[1 / b]$ which split completely in $Z[1 / b]$. Thus exactly as in the proof of Theorem 6.3 , it follows that the automorphism group of $Z[1 / b]$ acts faithfully on its spectrum. This implies that $\sigma$ is trivial on $Z$, as desired.

If $R$ is algebraic over $k$, then we can assume $k$ is a field and so $Z(R)$ is finite dimensional over $k$. Thus $\operatorname{Aut}_{k}(Z)$ is a finite group, $\sigma$ has finite order on $Z$ and (c) holds.

We can now prove an analog of the torsion result for commmutative rings. We first prove a type of nilpotence result for rank zero bimodules. This was originally proved by Bass for commutative noetherian rings. Wiegand [W] recently gave a simpler proof of Bass' result. His proof depended on localizations which we cannot use. Moreover, the result in our case is only true for bimodules which commute with primes. This distinction does not occur in the commutative case. Our proof in fact provides a new proof of the commutative result.

Theorem 3.4. Let $R$ be affine over $k$ and have Krull dimension $d$. Assume $k$ is either a field or the integers. If $X, X_{i}, Y_{i} \in \mathscr{J}_{n}$ for $0 \leq i \leq d$, then

(a) $\prod_{i=0}^{d}\left(X_{i}-Y_{i}\right)=0$ as left modules,

(b) $(X-R)^{d+1}=0$ as left $R$ modules, and

(c) $X^{m}$ is stably free as a left module for $m=n^{u}$ and $u>\log _{2}(d)$.

Proof. Clearly (a) implies (b). By Lemma 3.1, (b) implies (c). Thus it suffices to prove (a). By Nakayama's Lemma, we can assume that $R$ is semiprime. We induct on the Krull dimension $d$ of $R$ and on the number of minimal primes of $R$. If the dimension is zero, then $R$ is artinian and so $X_{0} \cong R \cong Y_{0}$ as left modules by the Krull-Schmidt Theorem. Now $R$ embeds in $R / P_{1} \oplus \cdots \oplus R / P_{m}$ where $\left\{P_{1}, \ldots, P_{m}\right\}$ is the set of minimal primes of $R$. Suppose $R / P_{m}$ has Krull dimension 0 . Then this quotient is artinian and splits off. So $R=R^{\prime} \oplus$ $R / P_{m}$ and the result follows by induction.

So we can assume that each $R / P_{i}$ has positive Krull dimension. By Theorem 3.3(a,b) and Lemma 3.2, we can find $R$-bimodule maps $\alpha: Y_{0} \rightarrow X_{0}$ and $\beta: X_{0} \rightarrow Y_{0}$ such that $\alpha \beta$ and $\beta \alpha$ are multiplication by a regular central element $c$ of $R$. Let $I$ be the radical of $c R$. If $c$ is a unit, then $X_{0} \cong Y_{0}$ as 
bimodules. Thus we can assume that $R / I$ has Krull dimension at most $d-1$. Note that $I U=U I$ for any $U \in \mathcal{J}$. Thus $U / I U$ is an invertible bimodule of $R / I$.

Thus by induction

$$
\prod_{i=1}^{d}\left(X_{i} / I X_{i}-Y_{i} / I Y_{i}\right)=0 .
$$

Let $M$ and $N$ denote the positive and negative terms respectively of

$$
\prod_{i=1}^{d}\left(X_{i}-Y_{i}\right)
$$

Thus by (3.4.1), we mean that $M / I M \cong N / I n$, as left $R$-modules. By Nakayama's Lemma, in fact $M / c M \cong N / c N$. Let $\gamma \in \operatorname{Hom}_{R}(M, N)$ induce this last isomorphism. Let $\delta \in \operatorname{Hom}_{R}(N, M)$ induce the inverse. These maps exist since $M, N$ are projective. Moreover, since $M, N$ are projective, there exist $\tau \in \operatorname{End}_{R}(N)$ and $\tau^{\prime} \in \operatorname{End}_{R}(M)$ with

$$
\gamma \delta=1+c \tau, \quad \delta \gamma=1+c \tau^{\prime} .
$$

Consider the left module maps

$$
\rho=\left(\begin{array}{ll}
1 \otimes \gamma & \alpha \otimes \tau \\
\beta \otimes 1 & 1 \otimes \delta
\end{array}\right)
$$

and

$$
\nu=\left(\begin{array}{cc}
1 \otimes \delta & -\alpha \otimes \tau^{\prime} \\
-\beta \otimes 1 & 1 \otimes \gamma
\end{array}\right)
$$

between $K=\left(X_{0} \otimes M\right) \oplus\left(Y_{0} \otimes N\right)$ and $L=\left(X_{0} \otimes N\right) \oplus\left(Y_{0} \otimes M\right)$. Note that these maps are well defined left module maps since each term is a tensor product of a bimodule homomorphism with a left module map.

It is straightforward to compute that $\rho \nu$ is an automorphism of $L$ and that $\nu \rho$ is an automorphism of $K$. Thus $K \cong L$ as left modules. This is equivalent to $\prod_{i=0}^{d}\left(X_{i}-Y_{i}\right)=0$ as left modules. This completes the proof.

The previous theorem showed that $\mathscr{J}_{n}$ is stably torsion of bounded exponent. We show that the same is true of $\mathscr{C}_{n} / \mathscr{Z}_{n}$ for $R$ noetherian. We recall that the pi degree of a prime pi ring $R$ is the smallest $n$ such that $R$ embeds in $M_{n}(C)$ for some field $C$. We say that $R$ has pi degree $f$ if $f$ is the maximum of the pi degrees of the prime images of $R$.

Theorem 3.5. Suppose $R$ is noetherian and semiprime of pi degree $f$. Let $T$ be the trace ring of $R$.

(a) $\mathscr{C}_{n} / \mathscr{J}_{n}$ acts faithfully on $\operatorname{spec}(R)$.

(b) Every orbit of $\mathscr{C}_{n}$ on $\operatorname{Spec}(T)$ and on $\operatorname{Spec}(R)$ has size at most $f$.

(c) If $X \in \mathscr{C}_{n}$, then $X^{e} \in \mathcal{J}$ for some e dividing $f$ !.

Proof. (a) follows by definition. (b) As in $\S 2$ if $X \in \mathscr{I}_{n}$, then $X$ induces an automorphism of $M_{n}(R)$. Let $T$ be the trace ring of $R$ (i.e., the direct product of the trace rings of $R / P, P$ a minimal prime). Thus $X$ induces an automorphism $\lambda$ of $M_{n}(T)$. Since $X$ is an ideal of $R$, it is $Z$-central. Thus $\lambda$ is trivial on $Z$ and so becomes inner on $M_{n}(Q)$. Thus $\lambda$ is also trivial on 
$C=Z(T)$. By [B], it follows that there are at most $f$ primes of $T$ over any prime of $C$. Thus $\mathscr{C}_{n}$ has orbits of size at most $f$ on $\operatorname{Spec}(T)$. Since $T$ is a module finite centralizing extension of $R$, this implies that $\mathscr{C}_{n}$ has orbits of size at most $f$ on $\operatorname{Spec}(R)$. Finally (c) follows from (a) and (b).

We remark that in the above result, we can avoid the reference to [B] by noting that $T$ is module finite over $C$ and so there are at most $m$ primes of $T$ over any prime of $C$ for some $m$. The result in [B] allows a more precise bound. We do not know whether the noetherian assumption is necessary.

Theorem 3.6. Let $k$ be a field or the integers. Let $R$ be a semiprime, noetherian pi ring which is affine over $k$. Assume that $R$ has pi degree $f$ and Krull dimension $d$. If $X$ is an invertible ideal of $R$ such that $n X \cong n R$ as left modules for some positive integer $n$, then $X^{m}$ is stably free as a left module for $m=\left(n^{u}\right) f$ ! and $u>\log _{2}(d)$.

Proof. This follows by Theorems 3.4 and 3.5.

If we replace $k$ by an arbitrary commutative noetherian domain, then a version of Theorem 3.6 is true with $m$ replaced by some positive integer. The proof is virtually the same except that when $R$ is algebraic over $k$, one must use Theorem 3.3(c).

Theorem 3.7. Let $k$ be a noetherian commutative domain. Assume $R$ is an affine pi $k$-algebra. Let $X$ be a $k$-central $R$ bimodule.

(a) If $X \in \mathcal{I}_{n}$, then there exists a positive integer $m$ such that $X^{m}$ is stably free as a left module.

(b) If $R$ is also semiprime and noetherian and $X \in \mathscr{I}_{n}$ is an invertible ideal of $R$, then there exists a positive integer $m$ such that $X^{m}$ is stably free as a left module.

Proof. By Theorem 3.5, it suffices to prove (a). We may assume that $R$ is semiprime by Nakayama's Lemma.

By Theorem 3.3(c), $X^{e}$ is isomorphic to an ideal of $R$ for some positive integer $e$. Replacing $X$ by $X^{e}$, we can assume that $X$ is an ideal of $R$. We follow the proof of Theorem 3.4. So choose bimodule maps $\alpha$ and $\beta$ and $c \in Z$ as in Lemma 3.1. By induction on Krull dimension, it follows that some power of $X / c X$ is stably free. We can replace $X$ by that power and assume that $X / c X$ is stably free (this will change the maps above and we will have to replace $c$ by a power of $c$ ). So there exist finitely generated free modules $F$ and $G$ such that $M=X \oplus F$ and $N=G$ are isomorphic modulo $c$. Choose homomorphisms $\gamma$ and $\delta$ which induce this isomorphism. Exactly as in the proof of Theorem 3.4 (using the same notation) we conclude that $K \cong L$. This implies that $X^{2} \oplus R$ is stably isomorphic (as a left module) to $X \oplus X$. By Lemma 3.1, the result follows.

Let $\mathscr{J}_{0, n}$ be the intersection of all the $\mathscr{J}_{n}$ conjugates of $\mathscr{J}^{s f} \cap \mathscr{J}_{n}$.

Corollary 3.8. Let $R$ be an affine pi $k$-algebra with $k$ a field or the rational integers.

(a) $\mathscr{J}_{n} /\left(\mathscr{L}_{0, n}\right)$ has exponent dividing $n^{u}$ for $u>\log _{2}(d)$.

(b) Assume $R$ is also semiprime and noetherian. Then $\operatorname{Picent}_{n}(R) /\left(\mathscr{J}_{0, n}\right)$ has exponent dividing $\left(n^{u}\right) f$ ! for $u>\log _{2}(d)$. 
Proof. Set $m=n^{u}$. By Theorem 3.4(c), if $X, U \in \mathscr{J}_{n}$, then $U X^{m} U^{-1}$ is stably free as a left module. This implies that the quotient group in (a) has exponent dividing $m$. Similarly (b) follows from Theorem 3.6.

The same proof using Theorem 3.7 instead of Theorem 3.6 yields

Corollary 3.9. Let $R$ be an affine pi k-algebra.

(a) $\mathscr{J}_{n} /\left(\mathscr{J}_{0}, n\right)$ is a torsion group.

(b) Assume $R$ is also semiprime and noetherian. Then $\operatorname{Picent}_{n}(R) /\left(\mathscr{J}_{0, n}\right)$ is a torsion group.

Thus we have an analog of the torsion result for commutative rings.

Lemma 3.10. Let $R$ be an affine semiprime noetherian pi ring over $k$ of Krull dimension $d$.

(a) $\mathscr{I}^{s f} \subseteq \mathscr{I}_{d+1}$.

(b) If $R=M_{v}(S)$ for $v>d$, then stably free can be replaced by free in Theorem 3.6.

Proof. By [MR, 6.7.4] $d+1$ is in the stable range of $R$. Then (a) holds by [MR, 11.7.13]. By Morita correspondence, (b) follows from (a).

Corollary 3.11. Let $X \in \mathscr{I}_{n}(R)$. If $R$ is module finite over $C=\{z \in Z \mid z x=$ $x z \forall x \in X\}$, then some power of $X$ is stably free as a left module.

Proof. By typical reduction arguments, we can assume that $C$ is finitely generated as a ring (and in particular is noetherian). Since there is a bound on the number of prime ideals of $R$ lying over any given prime of $C$, it follows that some power of $X$ is in $\mathcal{J}$. Now apply Theorem 3.6 .

We shall give an example below to show that stably free cannot be replaced by free. In the example, we produce invertible bimodules such that every nontrivial power is stably free but not free as a left module. We believe that the above results should also hold for a wider class of rings.

In the commutative case, there is a converse of the above results. Bass and the first author [BG] proved that (for $R$ commutative) an element $X$ of the Picent group has finite order if and only if $n X \cong n R$ for some positive integer $n$. This is not true in the noncommutative case. There exist orders over the integers with invertible ideals that are not locally free. Since the class group of invertible ideals is finite, every invertible ideal has finite order. However $n X \cong n R$ implies that $X$ and $R$ are locally isomorphic. One can prove a converse in the module finite situation by imposing the extra condition that the modules are locally free.

If we only consider bimodules of a special type, then the analog of the commutative result is true (and follows from the commutative result). Here we need no assumption on the ring.

Theorem 3.12. Let $R$ be a ring with 1. Let $Z$ be the center of $R$. Suppose $X$ is an $R$-bimodule such that $X$ is a bimodule summand of $m R$ for some $m$. Then $n X \cong m R$ as bimodules for some $m, n>0$ if and only $X^{\otimes e} \cong f R$ as bimodules for some $e, f>0$.

Proof. Let $D(R)$ denote the category of $R$-bimodules which are bimodule summands of $n R$ for some $n$. Note that $D(Z)$ is just the category of finitely 
generated projective $Z$-modules. Define $\phi: D(R) \rightarrow D(Z)$ by letting $\phi(X)$ be the set of bimodule homomorphisms from $R$ into $X$. It is straightforward to verify that $\phi$ is a bijection and preserves direct summands and tensor products. Thus the result follows by the same result for the commutative ring $Z$ (see $[\mathrm{BG}])$.

\section{The GROUP STRUCTURE OF Picent $n(R)$}

In this section, we investigate the group structure of $\operatorname{Picent}_{n}(R)$. In particular, we wish to see to what extent the abelian result for commutative rings can be recovered. By the examples in the next section, the best we can hope for is a stable version of the result.

For the rest of this section, let $k$ be a field (although there are versions of the results which hold more generally). Let $R$ be an affine semiprime pi $k$ algebra with center $Z$. By Lemma $3.2, \mathscr{C}=\operatorname{Picent}(R)$. We first study the stable behaviour of $\mathscr{J}_{n}$. If $G$ is a group, let $L_{i}(G)$ be the $i$ th term in the lower central series for $G$.

Theorem 4.1. Let $R$ have Krull dimension $d$. Then $L_{d}\left(\mathscr{J}_{n}\right) \subseteq \mathscr{S}$. Let $\mathscr{N}$ be the intersection of all $X\left(\mathscr{S} \cap \mathscr{J}_{n}\right) X^{-1}, X \in \mathscr{J}_{n}$. Then $\mathscr{N}$ is normal in $\mathscr{J}_{n}$ and is contained in $\mathscr{S}$ with the quotient $\mathscr{J}_{n} / \mathscr{N}$ a locally finite nilpotent group of exponent dividing $n^{u}$ where $u>\log _{2}(d)$.

Proof. We induct on $d$. If $d=0$, then $\mathscr{J}_{n} \subseteq \mathscr{C}_{1}$. So assume $d$ is positive. Exactly as in the proof of Theorem 3.4, we can assume that $R / P$ has positive Krull dimension for each minimal prime $P$.

Let $K_{0}(\mathscr{B})$ be as in Lemma 3.1. Let $T$ be the subring of $K_{0}(\mathscr{B})$ generated by the elements in $\mathscr{J}_{n}$. We can define a rank function on $T$ by declaring elements of $\mathscr{I}_{n}$ to have rank 1 . Let $U$ be the ideal of rank 0 elements. Let $\bar{T}=T / U^{d+1}$. Let $G=1+\bar{U}$. Thus $G$ is a subgroup of the unit group of $\bar{T}$. It is trivial to verify that $L_{d}(G)=1$. There is a homomorphism from $\mathscr{J}_{n}$ to the group $1+U$ sending $X \rightarrow R+(X-R)$. Composing this with the natural map from $T$ to $\bar{T}$ is a homomorphism from $\mathscr{J}_{n}$ to $G$. Let $L$ be the kernel of this map. Thus $L_{d}\left(\mathscr{Z}_{n}\right) \subseteq L$. Moreover, if $X \in L$, then viewed as an element of $T, X \in 1+U^{d+1}$. In particular, $X-R \in U^{d+1}$. Then by Theorem 3.4, $X-R$ annihilates $R$ considered as an element of $K_{0}(R)$. Thus $X$ is stably free as a left module. Hence $L \subseteq \mathscr{S}$. Thus $L_{d}\left(\mathscr{J}_{n}\right) \subseteq \mathscr{S}$ as desired. The last statement now follows from Corollary 3.8 and the fact that a nilpotent group of bounded exponent is locally finite.

In case $d=1, \mathscr{J}$ could be replaced by $\mathscr{C}$ by a slight modification of the proof of Theorem 5.2 below. We shall give an example to show that this fails for $d=2$. However, we do have some control over the quotient $\mathscr{C}_{n} / \mathscr{Z}_{n}$.

Theorem 4.2. Let $R$ be noetherian of pi-degree $f$ and Krull dimension $d$.

(a) $\mathscr{C}_{n} / \mathscr{J}_{n}$ is isomorphic to a subgroup of a finite direct product of symmetric groups of degree $f$.

(b) There exists a normal subgroup $\mathscr{N}$ of $\mathscr{C}_{n}$ with $\mathscr{N} \subseteq \mathscr{S}$ and $\mathscr{C}_{n} / \mathscr{N}$ locally finite with exponent dividing $\left(n^{u}\right) f !$ for $u>\log _{2}(d)$. If $f \leq 4$, this quotient is solvable. 
Proof. By Theorem 3.5, we know that $\mathscr{C}_{n} / \mathscr{J}_{n}$ acts faithfully on $\operatorname{Spec}(R)$ and that each orbit has size at most $f$. Since this quotient group is finite by Theorem 6.1 , (a) holds. In particular if $f \leq 4$, this quotient is therefore solvable. Now (b) follows by Theorem 4.1 and (a).

It seems likely that one can in fact remove the subscript $n$ in the previous theorem.

We now describe the promised example. Let $T$ be a Dedekind domain affine over $k$ such that $T$ contains $n$ nonzero ideals $W_{i}$ of order $n$ in the class group of $T$ such that for each $m, 1 \leq m \leq n$, the $W_{i}^{m}$ are pairwise nonisomorphic as $T$-modules. Let $U_{i}=T \oplus W_{i}$ for $1 \leq i<n$. Set $U=U_{1} \oplus \cdots \oplus U_{n}$. Let $T[x]$ be the polynomial ring over $T$, and set $V_{i}=U_{i}[x]$ and $V=U[x]$. Let $S=\operatorname{End}_{T[x]}(V)$. We can view $S$ as a ring of $n \times n$ matrices where the $(i, j)$ entry is a homomorphism from $V_{i}$ to $V_{j}$. Finally, we take $R$ to be the subring of $S$ consisting of all matrices where the off diagonal terms are multiples of $x$.

Similarly $M_{n}(S) \cong \operatorname{End}_{T[x]}(n V)$ can be identified with $n \times n$ matrices where the $(i, j)$ entry is a homomorphism from $n V_{i}$ to $n V_{j}$. Then $M_{n}(R)$ consists of those elements of $M_{n}(S)$ where the off diagonal terms are multiples of $x$.

By the choice of $W$, we know that $n V_{i}$ is free for each $i$. Thus $M_{n}(S)$ can be identified with $n \times n$ matrices over the ring $M_{2 n}(T[x])$, and $M_{n}(R)$ is the subring where all off diagonal terms are multiples of $x$. Thus conjugation by the permutation matrix which is the identity in all but the $i$ th and $j$ th rows and columns induces an inner automorphism $\sigma_{i j}$ of $M_{n}(S)$ and induces a central automorphism of $M_{n}(R)$. Let $\phi: T[x] \rightarrow T$ be the map sending $x$ to 0 . Then $\phi$ induces a homomorphism on $R$ and $M_{n}(R)$ with kernels $L$ and $M_{n}(L)$. Note that $R / L \cong E_{1} \oplus \cdots \oplus E_{n}$, where $E_{i}=\operatorname{End}_{T}\left(U_{i}\right)$ and similarly for $M_{n}(R / L)$. Thus there are $n$ primes over $x T[x]$. Note that $T[x]=Z(R)$. Clearly, $\sigma_{i j}$ interchanges the $i$ th and $j$ th primes. This shows that $\mathscr{C}_{n} / \mathscr{J}_{n}$ maps onto $S_{n}$, the symmetric group of degree $n$.

It is straightforward to verify that $M_{m}\left(E_{i}\right)$ and $M_{m}\left(E_{j}\right)$ are not isomorphic for $m<n$ and so there is no automorphism of $M_{m}(R)$ interchanging the various primes over $x T[x]$. It is obvious that $\mathscr{C}$ fixes all primes of $R$ which do not contain $x T[x]$ (each such prime is generated by central elements). It follows easily that $\mathscr{C} / \mathscr{J}$ acts faithfully on the primes of $R$ which are minimal over $x T[x]$. The above remarks show that $\mathscr{C}_{m}=\mathscr{J}_{m}$ for $m<n$ and that $\mathscr{C}_{n} / \mathscr{J}_{n} \cong S_{n}$. Since $R$ has Krull dimension $2, \mathscr{S} \subseteq \mathscr{C}_{3}$. In particular, if we take $n>3, \mathscr{S} \subseteq \mathscr{J}$. This shows that Theorem 4.1 is not true with $\mathscr{J}_{n}$ replaced by $\mathscr{C}_{n}$. We summarize the properties of the example in the next result.

Theorem 4.3. Let $k$ be a field. Let $n>3$. There exists a prime noetherian affine $k$-algebra $R$ of pi-degree $2 n$ and Krull dimension 2 such that $\operatorname{Picent}_{n}(R) / \mathscr{J}_{n} \cong$ $S_{n}$ and $\mathscr{J}_{n} \supseteq \mathscr{S}$. In particular, for $n>4, \operatorname{Picent}_{n}(R)$ is not "stably" solvable.

\section{ONE DIMENSIONAL RINGS}

In this section we will assume that $R$ is a prime ring (although much of what we say holds more generally) with an artinian quotient ring $Q$. If $R$ is commutative, then of course the group of invertible fractional ideals of $R$ is an abelian group (and in particular the group of principal invertible ideals is normal). This fails in the noncommutative case. Montgomery and Passman 
(see [M2]) constructed an example with $R$ an order in a quaternion algebra. In their example, Picent $(R)=\operatorname{Picent}_{2}(R) \cong S_{3} \times C_{2}$ and $\operatorname{Picent}_{1}(R) \cong C_{2} \times$ $C_{2}$. It follows from Lemma 5.4(a) below that in fact all $X \in \operatorname{Picent}(R)$ are stably free in this example. Here we show that in the one dimensional case the group of left stably free invertible ideals form a normal subgroup of the locally free invertible ideals and $\operatorname{Picent}_{n}(R)$ modulo this normal subgroup is abelian (indeed see Lemma 5.4 for a somewhat stronger statement).

Recall that $\mathscr{E}$ is the subgroup of the group of invertible bimodules generated by invertible ideals and $\mathscr{S}$ is the subgroup of $\mathscr{C}$ consisting of (left) stably free modules. Recall also that $\mathscr{C}_{n}=\mathscr{C} \cap \mathscr{I}_{n}$ and that by (3.2), for semiprime pi rings with only finitely many minimal ideals, $\mathscr{C}=$ Picent .

Lemma 5.1. Assume $X, Y \in \mathscr{C}$. If

(a) $R / X$ has finite length as a left module, and

(b) $n Y \cong n R$ as left modules, then

$$
X \oplus Y \cong(X \otimes Y) \oplus R
$$

as left modules.

Proof. Consider the short exact sequences of bimodules:

$$
0 \rightarrow X \rightarrow R \rightarrow R / X \rightarrow 0 \text {, }
$$

and

$$
0 \rightarrow X \otimes Y \rightarrow Y \rightarrow(R / X) \otimes Y \rightarrow 0 .
$$

The second sequence is exact since $Y$ is projective. We claim that $R / X \cong$ $(R / X) \otimes Y$ as left modules. Note that the left structure does not depend on the right structure of $Y$. Thus since $n Y \cong n R$, it follows that $n(R / X) \cong$ $n((R / X) \otimes Y)$ as left modules. Since these modules are of finite length, the claim follows. Now the result follows by Schanuel's Lemma.

Theorem 5.2. Assume $R$ is prime and every proper homomorphic image of $R$ is artinian. Then $\mathscr{C}_{n} /\left(\mathscr{S} \cap \mathscr{C}_{n}\right)$ is abelian of exponent $n$.

Proof. By the previous lemma, we know that for $X, Y \in \mathscr{C}_{n},(X Y \oplus R) \cong$ $(Y X \oplus R)$ as left modules. Thus $X Y$ and $Y X$ are stably isomorphic. This implies that $X Y X^{-1} Y^{-1} \in \mathscr{S}$. This shows that the quotient exists and is abelian. By the previous lemma and induction on $e$, we see that for $X \in \mathscr{C}_{n}$, $X^{e} \oplus(e-1) R \cong e X$ (as left modules). By taking $e=n$, we see that $X^{n} \in \mathscr{S}$, as desired.

In the classical case of orders, the previous result is well known. Moreover under mild assumptions (e.g., the Eichler or Drozd conditions, see [CR]) stably free modules are free and so $\mathscr{S}=\mathscr{C}_{1}$. It is possible that $\mathscr{S}$ is normal under much weaker hypotheses.

We want to compute these groups in a special example to show that stably free cannot be replaced by free in Theorem 3.6. For the rest of the section, fix a noetherian commutative domain $Z$ of Krull dimension 1 and let $R$ be a module finite prime $Z$-order. By Lemma 3.2, we have $\mathscr{C}=\operatorname{Picent}(R)$. Let LFPicent $(R)$ be the subgroup of Picent $R$ consisting of those elements which are locally free (as left and therefore as right modules). Note that LFPicent $(R)$ contains $\mathscr{S}$ and $\operatorname{Picent}_{n}(R)$ for all $n$. We recall some facts about such orders. Now Lemma 5.1 applies and in fact a minor variation of the proof yields the following well-known result. 
Lemma 5.3. If $X, Y \in \operatorname{LFPicent}(R)$, then $X \oplus Y \cong X Y \oplus R$ as left modules.

Let $L_{1}$ denote the locally free class group of $R$. Thus the elements of $\mathrm{LF}_{1}$ are stable isomorphism classes $[A]$ of left $R$-modules which are locally isomorphic to $R$. This is an abelian group via $[A]+[B]=[C]$ if and only if $A \oplus B \cong C \oplus R$. It also follows from this that if $A$ is a stably free rank one left $R$-module, then $A \oplus A \cong A \oplus R$ is free. In particular, $\mathscr{S} \subseteq \operatorname{Picent}_{n}(R)$ for all $n>1$. Now define $\theta: \operatorname{LFPicent}(R) \rightarrow \mathrm{LF}_{1}$ by $\theta(X)=[X]$. The previous remarks yield

Lemma 5.4. (a) $\theta$ is a homomorphism into the abelian group $\mathrm{LF}_{1}$ with $\operatorname{ker} \theta=$ $\mathscr{S}$.

(b) $X \in \operatorname{Picent}_{n}(R)$ for $n>1$ if and only if $\theta(X)$ has order dividing $n$.

(c) $\operatorname{Picent}_{n}(R)$ is normal in LFPicent $(R)$ for all $n>1$.

The previous results apply more generally to semiprime affine pi rings of Krull dimension 1 (with essentially no change in the proofs). Let Picent ${ }_{\infty}(R)=$ $\bigcup_{n}$ Picent $_{n}(R)$ and observe that if $R$ is module finite over its center, then Picent $_{\infty}(R) \subseteq$ LFPicent $(R)$. Since in the one dimensional case $\mathscr{S} \subseteq$ Picent $_{n}(R)$ for $n>1$, it follows from Lemma 5.4 that, for semiprime affine pi rings of Krull dimension 1, Picent ${ }_{n}(R), n>1$, is normal in Picent $\infty(R)$. We ask whether there is an analogous result for higher Krull dimension. In particular:

Question 5.5. If $R$ is a semiprime affine noetherian pi ring of Krull dimension $d$, is $\operatorname{Picent}_{n}(R), n>d$, normal in $\operatorname{Picent}_{\infty}(R)$ ?

Now choose a field $F$ which is not algebraic over a finite field and $\sigma \in \operatorname{Aut}(F)$ of order $n>1$. Let $k$ be the fixed field of $\sigma$. Let $T=F[x ; \sigma]$ be the skew polynomial ring. Now $Z=Z(T)=k[y]$ where $y=x^{n}-1$. Note that $T / y T \cong M_{n}(k)$ (since it is a cyclic algebra and 1 is a norm from $F$ to $k$ ). In particular, $y T$ is a maximal ideal of $T$.

Let $R=Z+y T$. Let $s \in T$ with $s$ a unit in $T / y T$. We define a left $R$-module $X(s)$ as follows: It is sufficient to describe it locally. If $P$ is any maximal ideal of $Z$ other than $y Z, X(s)_{P}=T_{P}=R_{P}$ and $X(s)_{y Z}=s R_{y Z}$. Also if $s-t \in y T$, then $X(s)=X(t)$. In fact, $X(s)$ is an $R$-bimodule. It suffices to determine this locally. Clearly this holds at every prime ideal of $Z$ except possibly $y Z$. Since $y T_{y Z}$ is the Jacobson radical of $T_{y Z}$, it follows that $s \in T$ is a unit in $T_{y Z}$ if and only if $s$ is a unit in $T / y T$. Hence for any such $s, s R_{y Z}=R_{y Z} s$, and we see that $X(s)$ is a bimodule. Note that in fact, $X(s)=T \cap R_{y Z} s=T \cap s R_{y Z}$. Moreover, $X(s)$ is invertible (with inverse $X(t)$ where $s t-1 \in y T)$ and is locally free by construction. Also by construction, $X(s) X(t)=X(s t)$. Thus there is a homomorphism $\alpha: G L_{n}(k)=(T / y T)^{*} \rightarrow$ LFPicent $(R)$ given by $\alpha(s)=X(s)$. If $X(s) \cong R$ as left modules, then $X(s)=$ $R t$ for some $t \in Q$, where $Q$ is the quotient ring of $T$. By the local conditions defining $X(s)$, we see that $t \in T^{*}=F^{*}$ and $s t^{-1} \in R_{y Z}^{*}=k^{*}+y R_{y Z}$ (see [G1]). It follows that $X(s)$ is free as a left module if and only if $s \in F^{*}$, when viewed as an element of $T / y T$. Moreover, since LFPicent $(R) / \mathscr{S}$ is abelian, it follows that the image of $S L_{n}(k)$ is in $\mathscr{S}$. Since there are elements in $S L_{n}(k)$ such that no nontrivial power is in $F^{*}$ (viewed as a subgroup of $G L_{n}(k)$ ), it follows that there exists $X \in \mathscr{S}$ with no power of $X$ free as a left module.

In fact, it is quite easy to see that if $X$ is an element of LFPicent $(R)$, then $X \cong x^{i} X(s)$ as bimodules for some $i \geq 0$ and some $s$. (To see this note that by 
Lemma 3.1, we can assume $X$ is an ideal of $R$. Then $T X \cong T$ as left modules implies $X \cong X(r)$ as left modules for some $r$. This implies $X=X(r) w$ for some $w$ in the quotient ring of $T$. Since $X$ is a bimodule, this implies $w$ is a normalizing element of $R$. It is straightforward to compute that up to units in $T$ the only normalizing elements are powers of $x$.) This also shows that there is a map from LFPicent $(R)$ to LFPicent $(T)$ (given by $X \rightarrow X T$ ). Since $X(s) \cong R$ as bimodules if and only if $s$ is a scalar $\bmod y T$ and $x^{i} R \cong R$ as bimodules if and only $n \mid i$, we have a short exact sequence of groups

$$
1 \rightarrow P G L_{n}(k) \rightarrow \operatorname{LFPicent}(R) \rightarrow C_{n} \rightarrow 1,
$$

where $C_{n}$ is the cyclic group of order $n$. It is straightforward to show this sequence splits. This shows that $\operatorname{LFPicent}(R)=\operatorname{Picent}_{n}(R) \cong P G L_{n}(k) \times$ $C_{n}$. One can also prove this using [CR, 55.30]. We summarize the preceding discussion.

Theorem 5.6. Let $F / k$ be a cyclic field extension of degree $n$ with $\sigma$ a generator of the Galois group of $F / k$. Let $T=F[x ; \sigma]$ be the skew polynomial ring. Let $Z$ be the center of $T$, and set $R=Z+y T$, where $y=x^{n}-1$.

(a) $\operatorname{LFPicent}(R)=\operatorname{Picent}_{n}(R) \cong P G L_{n}(k) \times C_{n}$.

(b) $\operatorname{Picent}_{1}(R)=\left(F^{*} / k^{*}\right) \times C_{n}$.

(c) $\mathscr{S} \geq P S L_{n}(k)$.

(d) If $k$ is not algebraic over a finite field, there exists $X \in \mathscr{S}$ such that $X^{i}$ is not free for any positive integer $i$.

A similar analysis shows that there exist prime orders over the integers which have stably free invertible bimodules of order greater than any preassigned integer (these orders must necessarily be contained in a totally definite quaternion algebra). Indeed, let $T$ be a maximal order over the integers in a totally definite quaternion algebra with $Z$ the center of $T$. Let $P$ be any unramified prime of $T$ such that $|T / P|$ is odd. Then $T / P \cong M_{2}(k)$, where $k=k(P)=Z /(P \cap Z)$. Set $R=Z+P T$. As above, we find that $\operatorname{LFPicent}(R)$ contains a subgroup $D$ isomorphic to $P G L_{2}(k)$ and that $\left|D \cap \operatorname{Picent}_{1}(R)\right| \leq\left|T^{*} / Z^{*}\right|$. Since the last term is finite, it follows that it is bounded independently of $P$. As the size of the residue field tends to infinity, we can find elements of arbitrarily large order in $P S L_{2}(k)$ and so elements of $\mathscr{S}$ with arbitrarily large order. This yields

Proposition 5.7. Given a positive integer $d$, there exists a prime order $R$ over the integers and an invertible bimodule $X$ of $R$ such that:

(a) $X \oplus R \cong R \oplus R$, as left modules, and

(b) $X^{i}$ is not a free left $R$-module for $0<i<d$.

This answers a portion of [GR2, (4.10)]. The example of Montgomery and Passman [M2] also yields an order with an invertible bimodule which is stably free but not free as a left module (the module has order 3 in the Picard group). It is still open whether sucli bimodules exist for integral group rings (see [GR2]).

\section{Automorphisms AND Spec}

In this section, we include some results of Braun and Vonessen and extend them. These results were used in previous sections. 
Theorem 6.1 [BV]. Let $R$ be an affine pi algebra over a field $k$, and let $G$ be a group acting on $R$ by $k$-algebra automorphisms. Assume that every prime ideal of $R$ has a finite $G$-orbit. Then there is a subgroup $H$ of $G$ of finite index stablizing each prime ideal of $R$. Moreover, if $R$ is semiprime, then $H$ can be chosen such that it fixes the center of $R$ elementwise.

This theorem extends a result of Farkas [F], who proved the result when $R$ was commutative and every ideal had a finite $G$-orbit. For its proof, we need the following lemma which has been independently obtained by Farkas.

Lemma 6.2 [BV]. Let $R$ be an affine commutative domain over a field $k$, and let $G$ be a group of $k$-algebra automorphisms of $R$. Assume that every height one prime ideal of $R$ has a finite $G$-orbit. Then $G$ is finite.

Proof. Using the principal ideal theorem, one sees that every prime ideal of $R$ is a finite sum of height-one prime ideals. Hence every prime ideal of $R$ has a finite orbit. Denote by $\bar{R}$ the integral closure of $R$. Then $G$ acts on $\bar{R}$. Since $\bar{R}$ is a finite $R$-module, over every prime ideal of $R$ there lie only finitely many prime ideals of $\bar{R}$. Hence also every prime ideal of $\bar{R}$ has a finite orbit. Replacing $R$ by $\bar{R}$, we may therefore assume that $R$ is integrally closed. We now show that every principal ideal of $R$ has a finite orbit. Then we apply Farkas' result to deduce that $G$ is finite.

Let $x$ be a nonzero element of $R$. Let $Q$ be a primary ideal associated with $x R$, and let $P$ be the radical of $Q$. Localize at $P$. The ring $R_{P}$ is an integrally closed local Noetherian domain with maximal ideal $P R_{P}$. Moreover, $P R_{P}$ is a prime associated to $x R_{P}$. So $P R_{P}$ has grade 1 [K, p. 67], and therefore height 1 [K, Theorem 95]. Hence $R_{P}$ is a discrete valuation ring. Denote by $H$ the stablizer of $P$ in $G$. Then $H$ has finite index in $G$. Since $R_{P}$ is a DVR, $Q R_{P}$ is a power of $P R_{P}$ and therefore also $H$-stable. Hence so is $Q=Q R_{P} \cap R$. It follows that every primary component of $x R$ has a finite $G$-orbit. Hence so does $x R$.

Proof of Theorem 6.1. Since $R$ has only a finite number of minimal prime ideals, it is easy to reduce to the case that $R$ is prime. We proceed by induction on the pi-degree $n$ of $R$. The case that $R$ is commutative has been dealt with by the lemma. So assume now that $R$ is an affine prime pi algebra of pi-degree $n \geq 2$. Denote by $T R$ the trace ring of $R$ and by $C$ the center of $T R$. The action of $G$ on $R$ extends to an action on $T R$. Since we have unique lying over between $\operatorname{Spec}_{n} R$ and $\operatorname{Spec}_{n} T R$, all prime ideals of pi-degree $n$ of $T R$ have a finite orbit. Since there are only a finite number of prime ideals of height 1 in $T R$ with pi-degree less than $n$, all height-one prime ideals of $T R$ have a finite orbit. Since $C \subseteq T R$ is a finite integral extension for which lying over and incomparability hold, also all prime ideals of $C$ of height 1 have a finite orbit. The lemma shows now the existence of a subgroup $H_{1}$ of $G$ of finite index such that $H_{1}$ acts trivially on $C$. In particular, $H_{1}$ fixes the center of $R$ pointwise. Since we have unique lying over for both $\operatorname{Spec}_{n} C$ with $\operatorname{Spec}_{n} T R$ and $\operatorname{Spec}_{n} R$ with $\operatorname{Spec}_{n} T R, H_{1}$ acts trivially on $\operatorname{Spec}_{n} R$. If $I$ is the ideal of $R$ generated by the evaluations of central polynomials for $(n-1) \times(n-1)$ matrices, then $G$ acts on $R / I$ and by induction there is a subgroup $\mathrm{H}_{2}$ of $G$ of finite index stablizing all prime ideals of $R / I$. Then $H=H_{1} \cap H_{2}$ is a subgroup of finite index in $G$ stabilizing all prime ideals of $R$ and fixing the center of $R$ elementwise. 
Using Lemma 6.2, we can show that automorphisms act faithfully on Spec. We also give a proof which does not depend on Farkas and Braun and Vonessen. Recall that a regular orbit for a group is an orbit such that no nonidentity element fixes a point. A $k$-algebra is called subaffine over $k$ if it is a subring of an affine $k$-algebra. It is not true in general that a homomorphic image of a subaffine ring is subaffine.

Theorem 6.3. Let $k$ be a field. Let $R$ be a commutative subaffine domain over $k$ of positive Krull dimension. Let $G$ be a group of $k$-algebra automorphisms of $R$.

(a) If $R$ has Krull dimension 1 and $G$ is finite, then $G$ has infinitely many regular orbits on $\operatorname{Spec} R$.

(b) $G$ acts faithfully on $\operatorname{Spec} R$.

Proof. We first prove (a). We can assume $R$ is integrally closed by inverting some element of the fixed ring $S=R^{G}$. Since $R$ is one dimensional, it is in fact affine (cf. [AS, Lemma 2.4]). Then $S$ is also affine. By [G2] (for $k$ infinite) or [J1], there are infinitely many primes of $S$ which split completely in $R$. Since $G$ acts transitively on all primes of $R$ over a given prime of $S$, the result follows.

We now prove (b). If $R$ has Krull dimension 1, the result follows from (a) and the fact that the group of $k$-automorphisms which act trivially on $\operatorname{Spec}(R)$ is finite by Lemma 6.2. Now let $\sigma$ be a $k$-automorphism of $R$ which acts trivially on $\operatorname{Spec} R$. By induction on Krull dimension, $\sigma$ acts trivially on $R / P$ for every height one prime $P$ such that $R / P$ is subaffine. There exists a regular central element $c$ of $R$ such that $R[1 / c]$ is affine. Then $R / P$ is subaffine for any prime $P$ not containing $c$. Since the intersection of all height one primes of $R[1 / c]$ is 0 , it follows that $\sigma$ is trivial on $R$.

We give another proof for $k$ infinite which does not use Lemma 6.2. Just as above it suffices to assume $R$ has Krull dimension 1 and so s affine. Thus $R$ is integral over $k[t]$. By passing to the subring $R^{\prime}$ of $R$ of separable elements over $k(t)$, we can assume $R=R^{\prime}$ (if $\sigma$ is trivial on $R^{\prime}$, it is trivial on $R$ ). Let $Q$ be the quotient field of $R$. Choose a generator $\theta \in R$ for $Q / k(t)$. By [G2], there are infinitely many primes $P_{\alpha}, \alpha \in k$, such that $\theta-\alpha \in P_{\alpha}$. Thus $\sigma(\theta)-\theta \in P_{\alpha}$. Since the intersection of any infinite set of height one primes is zero, it follows that $\sigma$ is the identity.

In fact, using induction one can show that $G$ acts faithfully on height one primes (or equivalently primes of fixed positive height). One can show that if $G$ is finite, then $G$ will have infinitely many regular orbits on primes of a given height (see [J2]). A version of the previous result is also true for $k$ the ring of rational integers. We now apply the previous results to pi rings.

Theorem 6.4. Let $R$ be a semiprime affine pi ring over the field $k$. Assume $R / P$ has positive Krull dimension for every minimal prime $P$. If $G$ is a group of $k$-algebra automorphisms of $R$ which acts trivially on $\operatorname{Spec} R$, then $G$ is trivial on the center of $R$.

Proof. Again, as in the proof of Theorem 6.1, we can reduce to the case $R$ is prime. We follow the notation of that proof. So $C=Z(T R)$ is affine. It follows that $G$ fixes all but finitely many height one primes of $C$. Thus there 
is a subgroup $H$ of finite index which fixes all primes of $C$ and hence by the previous result acts trivially on $C$. Thus we can invert some element $c$ of $C^{G}$ so that $G$ fixes all primes of $C[1 / c]$. Hence $R$ is trivial on $C[1 / c]$ by Theorem 6.3 and so on the center of $R$.

There is an easy proof of a slightly different version of Lemma 6.2 when $k$ is uncountable.

Proposition 6.5. Let $k$ be an uncountable field. Let $R$ be a commutative affine domain over $k$. If $G$ is a group of $k$-automorphisms of $R$ such that every orbit of $G$ is finite on $\operatorname{Max}(R)$, then $G$ is finite.

Proof. First note that we may assume that $k$ is algebraically closed. (Tensor with the algebraic closure. This ring has only finitely many minimal primes. Pass to a subgroup of finite index stablizing all the minimal primes, and then pass to a prime homomorphic image. Since only finitely many maximal ideals of the extension contract to the same ideal, the hypothesis is still valid.) We induct on the Krull dimension of $R$. If the dimension is 0 , there is nothing to prove. Let $V_{n}$ be the set of maximal ideals whose $G$-orbit has size at most $n$. Since $k$ is uncountable, no variety of positive dimension over it is a countable union of proper subvarieties. Thus $V_{n}$ is dense in $V=\operatorname{Max}(R)$ for some $n$. We claim $G$ has order at most $n$. It suffices to assume that $G$ is finitely generated. Let $N$ be the normal subgroup of $G$ which is the intersection of all subgroups of index at most $n$. Since $G$ is finitely generated, $N$ has finite index in $G$. Since $N$ fixes the dense subset $V_{n}$ of $V, N=1$, and so $G$ is finite. Since the field is algebraically closed, all orbits of unramified points are regular. Since the set of ramified points is contained in a proper subvariety, there must be an unramified point in $V_{n}$, and the result follows.

Note that the above result fails for $k$ a finite field. If $R=k[x, y]$, then $G=\operatorname{Aut}_{k}(R)$ has finite orbits on the maximal ideals of $R$ but clearly $G$ is not finite.

\section{The Weyl Algebra AS A FIXEd RING}

Recently S. P. Smith [Sm] has proved that the (first) Weyl algebra $A$ over C cannot be a fixed ring $S^{G}$, for $G$ a finite solvable group and $S \supset A$ a C-domain such that $S$ is finitely generated as a left or right $A$-module. Here we improve his result to finite groups $G$ such that $G$ is not perfect (that is $G \neq[G, G])$. We shall actually prove that under fairly mild assumptions the existence of a nonperfect extension implies the existence of a cyclic extension. We then reprove Smith's result for the cyclic case for a class of simple noetherian domains.

Theorem 7.1. Let $A$ be a simple domain with algebraically closed center $k$. Let $S$ be a k-algebra containing $A$. If $S$ is a domain and $S^{G}=A$ for $G$ a finite nonperfect subgroup of $\operatorname{Aut}_{k}(S)$ with $|G|^{-1} \in k$, then

(i) $Z(S)=k$.

(ii) $G$ consists of outer automorphisms.

(iii) There exists a $k$-domain $B$ and a cyclic nontrivial homomorphic image $H$ of $G$ such that $B^{H}=A$. Moreover, $B$ is simple and finitely generated as a left and right module and is an A-bimodule summand of $S$. 
Proof. Set $Z=Z(S)$. Then $Z^{G}=k$. Thus $Z$ is algebraic over $k$. Since $k$ is algebraically closed, this implies $Z=k$ and (i) holds.

Next note that $S$ is simple, since any nonzero ideal of $S$ intersects $A$ by the Bergman-Isaacs theorem (cf. [M1, 1.5]) and $A$ is simple. Next, $G$ is outer on $S$. For, if $g \in G$ is inner, let $u \in S$ be such that $g(s)=u^{-1} s u \forall s \in S$. Then since $G$ is finite, $u^{n}$ is central for some $n$. Thus $u^{n} \in k$. Since $k$ is algebraically closed and $k[u]$ is a domain, this implies $u \in k$ and so $g=1$. Thus (ii) holds.

At this point we give two different proofs of (iii).

I. Since $S$ is simple, $G$ is outer, and the trace map $S \rightarrow S^{G}=A$ is surjective, we may apply the Galois correspondence theorem (based on the work of Kharchenko, cf. [MP, Theorem 12]) to obtain a bijection between subgroups $L$ of $G$ and intermediate rings $S^{L}$ of $S$. In particular if $G$ is not perfect, then there exists a proper normal subgroup $L$ of $G$ with $H=G / L$ cyclic. Set $B=S^{L} \neq A$. Then $B^{H}=S^{G}=A$. By the Bergman-Isaacs theorem, it follows that any nonzero ideal of $B$ must intersect $A$. Thus $B$ is simple. It remains only to show that $B$ is finitely generated as a left and right $A$-module. Since $H$ is cyclic and $|H| \neq 0$ in $k, B$ is the direct sum of the $H$ eigenspaces $B_{\lambda}=\{b \in B \mid h(b)=\lambda(h) b \forall h \in H\}$, where $\lambda$ is a character of $H$. Assume $B_{\lambda} \neq 0$. Then since $B$ is a domain, no power of it is nonzero. In particular, if $\lambda$ has order $t$, then $B_{\lambda}^{t}$ is a two sided ideal of $A$ and hence equals $A$. It follows easily from this that $B_{\lambda}$ is finitely generated as a left or right $A$-module and hence so is $B$. Moreover, since the order of $L$ is invertible, the trace map shows that $B$ is a bimodule summand of $S$.

II. We give another proof under the additional assumption that $S$ has a quotient division ring $D$ (e.g., if $S$ is noetherian). Then $G$ extends uniquely to a group of automorphisms of $D$. Moreover, $G$ remains outer on $D[\mathrm{M} 1$, proof of Example 3.8]. Also $D=S A^{-1}$ [M1, Theorem 5.3].

Now by a classical theorem of Nakayama on division rings, $D$ has a "normal basis" over $D^{G}$; that is $D$ is a free $D^{G}[G]$-module of rank one. Since the regular representation of $G$ appears, every irreducible representation of $G$ appears as component of $D$ over $D^{G}$.

In particular, $G$ not perfect implies that $G$ has a nontrivial degree one character $\lambda$. Then $D_{\lambda} \neq 0$, where

$$
D_{\lambda}=\{d \in D \mid g(d)=\lambda(g) d, \forall g \in G\} \text {. }
$$

Since $D=S A^{-1}, D_{\lambda}=S_{\lambda} A^{-1}$. In particular $S_{\lambda} \neq 0$. Now make $B=\Sigma S_{\lambda^{i}}$ and argue as above.

We now consider the cyclic case.

Theorem 7.2. Let $A$ be a simple noetherian domain satisfying the following conditions:

(i) $k=Z(A)$ is algebraically closed.

(ii) Every unit of $A$ is central.

(iii) If $X$ is a torsion element of $\operatorname{Picent}(A)$, then $X \in \operatorname{Picent}_{1}(A)$.

Then there exists no $k$-domain $S$ such that $S^{G}=A$ where $G$ is a finite nonperfect subgroup of $\operatorname{Aut}_{k}(S)$ and $|G| \neq 0$ in $k$.

Proof. By Theorem 7.1, we can take $G$ cyclic and $S$ finitely generated as a left and right $A$-module. Let $S_{\lambda}=I \neq 0$ be as above with $\lambda$ a nontrivial character. 
As in the proof of Theorem 7.1, we find that there exists an $A$-bisubmodule $J$ of $S$ such that $I J=J I=A$. Thus as above, $I$ is finitely generated as a left or right $A$-module. By a theorem of Goodearl [Br, Theorem 10], $I$ is projective as a left or right $A$-module. We claim that $I \in \operatorname{Picent}(A)$. The multiplication map yields a bimodule homomorphism from $I \otimes J$ onto $A$. Since $S$ is a domain, $A \supset I a \cong I$ as left $A$-modules and similarly as a right $A$-module. Thus $I$ and $J$ are rank one projective left and right $A$-modules. Thus this homomorphism is a bijection and $I$ is invertible. Since $I$ commutes with $k$, $I \in \operatorname{Picent}(A)$ as desired. As in the proof of Theorem 7.1, $I$ has finite order. Thus by assumption $I$ is free as a left and right module. So $I=A u=u A$ for some $u \in S$. Thus $u^{n} A=A=A u^{n}$ for some $n>0$. Hence $u^{n}$ is a unit of $A$ and thus is in $k$. Since $k$ is algebraically closed, this implies $u$ is in $k$ and so $I=A$, a contradiction.

Corollary 7.3. Let $A$ be the (first) Weyl algebra over $\mathrm{C}$ and let $S \supset A$ be a C-algebra such that $S$ is a domain. If $G$ is a finite group of automorphisms of $S$ such that $S^{G}=A$, then $G$ is perfect.

Proof. By Stafford [S], every element of $\operatorname{Picent}(A)$ is free. The center of $A$ is C. By a degree argument, every unit of $A$ is central. Thus the previous theorem applies and $G$ is perfect.

The question remains open for arbitrary finite groups. Indeed, as Smith observed, it is possible that $A$ cannot be properly contained in any domain which is finitely generated as a left and right module. As a partial result, we can show that with $A$ the Weyl algebra and notation as above $S$ has a normal basis over $S^{G}$. It follows that $S$ must have the structure $A \#_{\sigma} \mathbf{C}[G]^{*}$, a Hopf crossed product of the dual $\mathrm{C}[G]^{*}$ over $A$; however the summands are not $A$-bimodules when $G$ is not abelian. It is easy to show that a minimal counterexample to the theorem would be a simple group. However, we cannot prove the result even for $A_{5}$.

We close by noting that the previous corollary also applies to any domain $B$ Morita equivalent to $A$, the first Weyl algebra. We cannot apply Theorem 7.2 directly (see $[\mathrm{S}]$ ). However, suppose that $B=T^{G}$ for some group of automorphisms $G$. Extend $G$ to $R=M_{d}(T)$. Then $R^{G}=M_{d}(B)$. Since $A$ is Morita equivalent to $B, A=e M_{d}(B) e$ for some positive integer $d$ and some idempotent $e$. Set $S=e M_{d}(T) e$. Since $e \in R^{G}, G$ acts on $S$. Clearly, $S^{G}=e M_{d}(B) e=A$. Moreover, since $A$ and $B$ are domains, $e$ is a rank one idempotent and so $S$ is a domain. Now we can apply the previous corollary. See [Sm2] for an example of such a domain.

\section{REFERENCES}

[AS] M. Artin and W. Schelter, Integral ring homomorphisms, Adv. Math. 39 (1981), 289-329.

[BG] H. Bass and R. M. Guralnick, Projective modules with free multiples and powers, Proc. Amer. Math. Soc. 96 (1986), 207-208.

[B] A. Braun, An additivity principle for p.i. rings, J. Algebra 96 (1985), 433-441.

[BV] A. Braun and N. Vonessen, Some notes on a theorem of Farkas, preprint.

[Br] K. Brown, The representation theory of Noetherian rings, Noncommutative Rings, Math. Sci. Res. Inst. Publ., vol. 24, Springer-Verlag, Berlin and New York, 1992.

[CR] C. Curtis and I. Reiner, Methods of representation theory with applications to finite groups and orders. II, Wiley, New York, 1987. 
[F] D. Farkas, Groups acting on affine algebras, Trans. Amer. Math. Soc. 310 (1988), 485-497.

[G1] R. M. Guralnick, The genus of a module. II: Roiter's theorem, power cancellation, and extension of scalars, J. Number Theory 26 (1987), 149-165.

[G2] _ A question of Stafford about affine pi algebras, Comm. Algebra 18 (1990), 3055-3057.

[G3] _ Bimodules over pi rings, Methods in Module Theory, Marcel Dekker, New York, 1992, pp. 117-134.

[GR1] W. H. Gustafson and K. Roggenkamp, Automorphisms and Picard groups of hereditary orders, Texas Tech. Univ. Math. Ser. 15 (1987), 37-52.

[GR2] __ A Mayer-Vietoris sequence for Picard groups, with applications to integral group rings of dihedral and quaternion groups, Illinois J. Math. 32 (1988), 375-406.

[J1] Bao-Ping Jia, Splitting of rank one valuations, Comm. Algebra 19 (1991), 777-794.

[J2] _ Splitting of primes in extension domains, Comm. Algebra 19 (1991), 2603-2623.

[K] I. Kaplansky, Commutative rings, Univ. of Chicago Press, Chicago, 1974.

[KO] M. Knus and M. Ojanguran, Théorie de la descente et Algèbres d'Azumaya, Lecture Notes in Math., vol. 389, Springer-Verlag, Berlin and New York, 1974.

[MR] J. C. McConnell and J. C. Robson, Noncommutative Noetherian rings, Wiley, New York, 1987.

[M1] S. Montgomery, Fixed rings of finite automorphism groups of associative rings, Lecture Notes in Math., vol. 818, Springer-Verlag, Berlin and New York, 1980.

[M2] — A generalized Picard group for prime rings, Banach Center Publ. 26, Part I, PWNPolish Scientific Publishers, Warsaw, pp. 55-63.

[MP] S. Montgomery and D. Passman, Outer galois theory of prime rings, Rocky Mountain J. Math. 14 (1984), 305-318.

[RZ] A. Rosenberg and D. Zelinsky, Automorphisms of separable algebras, Pacific J. Math. 11 (1961), 1109-1117.

[Sm] S. P. Smith, Can the Weyl algebra be a fixed ring, Proc. Amer. Math. Soc. 107 (1989), 587-589.

[Sm2] _ An example of a ring Morita equivalent to the Weyl algebra $A_{1}$, J. Algebra 73 (1981), 552-555.

[S] J. T. Stafford, Endomorphisms of right ideals of the Weyl algebra, Trans. Amer. Math. Soc. 299 (1987), 623-639.

[W] R. Wiegand, Nilpotent elements in Grothendieck rings, Illinois J. Math. 32 (1988), 246-262.

Department of Mathematics, University of Southern California, Los Angeles, CaliFORNIA 90089-1113

E-mail address: guralnic@mtha.usc.edu

E-mail address: smont gom@mtha.usc.edu 